\title{
Hybrid dynamical electroweak symmetry breaking with heavy quarks and the 125 GeV Higgs
}

\author{
Michael Geller,, , $\rightarrow$ Shaouly Bar-Shalom,, , 团 and Amarjit Soni ${ }^{2}$, 田 \\ ${ }^{1}$ Physics Department, Technion-Institute of Technology, Haifa 32000, Israel \\ ${ }^{2}$ Theory Group, Brookhaven National Laboratory, Upton, NY 11973, USA
}

(Dated: October 18, 2018)

\begin{abstract}
Existing models of dynamical electroweak symmetry breaking (EWSB) find it very difficult to get a Higgs of mass lighter than $m_{t}$. Consequently, in light of the LHC discovery of the $\sim 125 \mathrm{GeV}$ Higgs, such models face a significant obstacle. Moreover, with three generations those models have a superheavy cut-off around $10^{17} \mathrm{GeV}$, requiring a significant fine-tuning. To overcome these twin difficulties, we propose a hybrid framework for EWSB, in which the Higgs mechanism is combined with a Nambu-Jona-Lasinio mechanism. The model introduces a strongly coupled doublet of heavy quarks with a mass around $500 \mathrm{GeV}$, which forms a condensate at a compositeness scale $\Lambda$ about a few $\mathrm{TeV}$, and an additional unconstrained scalar doublet which behaves as a "fundamental" doublet at $\Lambda$. This "fundamental"-like doublet has a vanishing quartic term at $\Lambda$ and is, therefore, not the SM doublet, but should rather be viewed as a pseudo-Goldstone boson of the underlying strong dynamics. This setup is matched at the compositeness scale $\Lambda$ to a tightly constrained hybrid two Higgs doublet model, where both the composite and unconstrained scalars participate in EWSB. This allows us to get a good candidate for the recently observed $125 \mathrm{GeV}$ scalar which has properties very similar to the Standard Model Higgs. The heavier (mostly composite) CP-even scalar has a mass around $500 \mathrm{GeV}$, while the pseudoscalar and the charged Higgs particles have masses in the range $200-300 \mathrm{GeV}$.
\end{abstract}

\section{INTRODUCTION}

With the recent LHC discovery of the $125 \mathrm{GeV}$ scalar particle [1], we are one step closer to understanding the mechanism of EWSB. The Standard Model (SM) Higgs mechanism lacks a fundamental explanation and has, therefore, been long questioned. Indeed, inspired from our experience with QCD, it has been speculated that the scalar responsible for EWSB may not be fundamental but, instead, some form of a fermion-antifermion bound state, which is generated dynamically by some new strong interaction at a higher scale [2].

The "conventional" models for this scenario are based on the Nambu-Jona-Lassinio (NJL) mechanism [3], in which Dynamical EWSB (DEWSB) is triggered by the condensation of heavy fermions. However, such models have one major caveat: the typical mass of the heavy fermionic condensate, $H \sim\langle\bar{\Psi} \Psi\rangle$, tends to lie in the range $m_{\Psi}<m_{H}<2 m_{\Psi}$ (see e.g., [4]). Thus, such a composite tends to be too heavy to account for the recently discovered $125 \mathrm{GeV}$ Higgs-like particle. ${ }^{[1]}$ Because of this difficulty, in this paper we propose an alternative solution for the $\mathrm{TeV}$-scale DEWSB scenario, in which a light SM-like Higgs with a mass of $\mathcal{O}\left(m_{W}\right)$ emerges. We

\footnotetext{
*Electronic address: mic.geller@gmail.com

${ }^{\dagger}$ Electronic address: shaouly@physics.technion.ac.il

${ }^{\ddagger}$ Electronic address: adlersoni@gmail.com

[1] One possible way out, which we will not consider here, is that the lightest scalar state is the pseudoscalar associated with DEWSB (see e.g., [5]), since its mass does not receive large corrections from loops of the heavy fermions and, thus, can in principle be held small without fine-tuning.
}

basically construct, as we will elaborate later, a hybrid DEWSB setup by adding an unconstrained scalar field at the compositeness scale, which behaves essentially like a "fundamental" field.

Indeed, one of the early attempts in this direction investigated the possibility of using the top-quark as the agent of DEWSB via top-condensation [4], in a generalization of the NJL model. However, the resulting dynamical top mass turns out to be appreciably heavier than $m_{t}$, thus making it difficult for top condensation to provide a viable picture. Moreover, top-condensate models [or NJL models where the condensing fermions have masses of $\left.\mathcal{O}\left(m_{t}\right)\right]$, require the cutoff for the new strong interactions to be many orders of magnitudes larger than $m_{t}$, i.e., of $\mathcal{O}\left(10^{17}\right) \mathrm{GeV}$, resulting in a severely fine-tuned picture of DEWSB.

In passing, we should mention that several interesting generalizations of the top-condensate model of [4], which potentially avoid these obstacles, have been suggested. For example, one can relax the requirement that only the top-condensate is responsible for the full EWSB [6, 7], or assume that condensations of new heavier quarks and/or leptons drive EWSB [8-12]. In such scenarios the resulting low-energy (i.e., EW-scale) effective theory may contain more than a single composite Higgs doublet [5, 7, 9-13]. Indeed, low-energy multi-Higgs models, with new heavy fermions with masses of $\mathcal{O}(500 \mathrm{GeV})$, are natural outcomes of a TeV-scale DEWSB scenario, since the heavy fermions are expected to be strongly coupled at the near by $\mathrm{TeV}$-scale and to lead to the formation of several condensates - possibly with sub-TeV masses.

In this paper, as alluded to above, we take another direction constructing a hybrid DEWSB setup by adding an unconstrained scalar field at the compositeness scale 
which behaves as a "fundamental" field where additional super-critical attractive 4-Fermi operators form a composite scalar sector. The "fundamental" scalar is unconstrained at the compositeness scale and may result from the underlying strong dynamics [6], e.g., it can be the pseudo-Goldstone boson of a global symmetry breaking at the strong interaction scale. This strongly coupled composite-plus-fundamental sectors are matched at the compositeness scale to a hybrid "4th generation" 2HDM (h4G2HDM), with one fundamental-like field $\left(\Phi_{\ell}\right)$ which couples to the SM's light fermions and one auxiliary (composite) field $\left(\Phi_{h}\right)$ which couples to the heavy quarks. ${ }^{[2]}$ The fundamental or unconstrained Higgs field is thus responsible for the mass generation of the lighter SM fermions and for the observed CKM flavor pattern.

We stress that the name "4th generation" is used here for convenience only and should not be confused with the minimal SM4 framework, as we explicitly involve a 2HDM. Furthermore, the DEWSB mechanism proposed here can be generalized to the case of non-sequential heavy quarks, e.g., new heavy vector-like quarks [15] (see further discussion in the summary). Later in this paper, we will briefly explore the phenomenology and study the confrontation of this model with the latest LHC data as well as flavor and EW precision constraints.

Our h4G2HDM setup is motivated by the concept that new heavy fermions are expected to have purely dynamical masses, while a different mechanism is expected to underly the generation of mass for the lighter SM fermions. It further allows us to get a light $125 \mathrm{GeV}$ SM-like Higgs, since its mass is mostly proportional to the quartic coupling of the fundamental field, which is unconstrained at the compositeness scale. That is, with this assignment, in our h4G2HDM the mass of the lightest CP-even Higgs state does not receive the usual large quantum corrections from loops of the heavy dynamical fermions, which instead feed into the quartic coupling of the composite scalar.

It should be noted that the simplest low-energy effective setup which may result from a TeV-scale DEWSB scenario is the so called SM4, i.e., the SM with a 4th sequential generation of heavy fermions and one Higgs doublet. This minimal framework has, however, several drawbacks [16] and, more importantly, it fails to account for the existence of the recently discovered $125 \mathrm{GeV}$ Higgs-like particle [17]. On the other hand, a 4th generation framework with two (or more) Higgs doublets, such as the h4G2HDM discussed here, where the new heavy quarks have masses of $\mathcal{O}(500 \mathrm{GeV})$, is not only consistent with the current Higgs data ${ }^{[3]}$, but it can also have

[2] Other interesting hybrid multi-Higgs model were suggested in 12, 14]. In particular, in [12] the condensates which drive DEWSB are formed by exchanges of the fundamental Higgs, while in [14] a hybrid setup similar to ours was constructed in the top-condensation scenario.

[3] For discussions on the phenomenology of multi-Higgs 4th gener- specific flavor structures, which may give rise to new signatures of 4th generation quarks at the LHC [16, 18], and substantially relax the current bounds on their masses [19]. Moreover, the lightest Higgs state in these class of models may be a good candidate for the recently discovered $125 \mathrm{GeV}$ Higgs-like particle [20].

\section{DYNAMICAL EW SYMMETRY BREAKING WITH HEAVY FERMIONS: A HYBRID SETUP}

We assume that the 4 th generation quarks are charged under some new strong interaction that dynamically breaks EW symmetry (see e.g., the "top-color" models of [6, 22]). The theory at the compositeness scale, $\Lambda$, can then be parameterized by adding to the light SM degrees of freedom the following set of strongly coupled 4-Fermi terms:

$$
\begin{aligned}
\mathcal{L}=\mathcal{L}_{S M}(\Lambda) & +G_{t^{\prime}} \bar{Q}_{L}^{\prime} t_{R}^{\prime} \bar{t}_{R}^{\prime} Q_{L}^{\prime}+G_{b^{\prime}} \bar{Q}_{L}^{\prime} b_{R}^{\prime} \bar{b}_{R}^{\prime} Q_{L}^{\prime} \\
& +G_{t^{\prime} b^{\prime}}\left(\bar{Q}_{L}^{\prime} b_{R}^{\prime} \bar{t}_{R}^{\prime c} i \tau_{2} Q_{L}^{\prime c}+\text { h.c. }\right)
\end{aligned}
$$

where $Q_{L}^{\prime}=\left(t_{L}^{\prime} \quad b_{L}^{\prime}\right)^{T}$ and $\mathcal{L}_{S M}(\Lambda)$ stands for the bare SM Lagrangian with a single fundamental Higgs field, $\Phi_{\ell}$, which essentially parameterizes our ignorance as for the origin of mass for the lighter fermions. It is given by:

$$
\begin{aligned}
& \mathcal{L}_{S M}(\Lambda)=\left|D_{\mu} \Phi_{\ell}\right|^{2}+\mathcal{L}_{S M}^{Y}(\Lambda)-V_{S M}(\Lambda), \\
& \mathcal{L}_{S M}^{Y}(\Lambda)=g_{u}^{0, i j} \bar{Q}_{L}^{i} \tilde{\Phi}_{\ell} u_{R}^{j}+g_{d}^{0, i j} \bar{Q}_{L}^{i} \Phi_{\ell} d_{R}^{j}+h . c . \\
& V_{S M}(\Lambda)=\left(\mu_{\ell}^{0}\right)^{2} \Phi_{\ell}^{\dagger} \Phi_{\ell}+\frac{1}{2} \lambda_{\ell}^{0}\left(\Phi_{\ell}^{\dagger} \Phi_{\ell}\right)^{2},
\end{aligned}
$$

where $\tilde{\Phi} \equiv i \tau_{2} \Phi^{\star}, i, j=1-3$ and we use the superscript 0 to denote bare couplings at the scale $\Lambda$.

We can reproduce the theory defined by Eq. 11 by introducing at $\Lambda$ an auxiliary Higgs doublet, $\Phi_{h}$, which couples only to the 4 th generation quarks as follows:

$$
\begin{aligned}
\mathcal{L}_{q^{\prime}}(\Lambda) & =g_{b^{\prime}}^{0}\left(\bar{Q}_{L}^{\prime} \Phi_{h} b_{R}^{\prime}+\text { h.c. }\right)+g_{t^{\prime}}^{0}\left(\bar{Q}_{L}^{\prime} \tilde{\Phi}_{h} t_{R}^{\prime}+\text { h.c. }\right) \\
& -\left(\mu_{h}^{0}\right)^{2} \Phi_{h}^{\dagger} \Phi_{h} .
\end{aligned}
$$

For simplicity we did not write above the Yukawa terms for the light and for the 4th generation leptons. In particular, our results are not sensitive to the choice by which the 4th generation leptons couple to the Higgs sector; they can either couple to the fundamental Higgs or to the auxiliary field. In either case, we assume that their couplings are sub-critical and, therefore, do not play any role in DEWSB (see also discussion below).

The scalar sector of the full theory at the compositeness scale is, therefore, described by:

$$
\mathcal{L}(\Lambda)=\mathcal{L}_{S M}(\Lambda)+\mathcal{L}_{q^{\prime}}(\Lambda)+\left(\mu_{h \ell}^{0}\right)^{2}\left(\Phi_{h}^{\dagger} \Phi_{\ell}+\text { h.c. }\right)
$$

ation models, see e.g., [13, 16, 18 21]. 
where we have added a $\Phi_{h}-\Phi_{\ell}$ mixing term $\left(\propto \mu_{h \ell}^{0}\right)^{2}$, which may arise e.g., from QCD-like instanton effects associated with the underlying strong dynamics (see e.g., 6, 23]) or from sub-critical couplings of the fundamental Higgs to the 4th generation quarks (see below). This term explicitly breaks the $U(1)$ Peccei-Quinn (PQ) symmetry [24], which is otherwise possessed by the model, thus avoiding the presence of a massless pseudoscalar in the spectrum. Note that, in any realistic scenario we expect $\mu_{h \ell}\left(\mu \sim m_{W}\right) \sim \mathcal{O}\left(m_{W}\right)$ and, since this is the only term which breaks the PQ symmetry, it evolves only logarithmically under the RGE so that, at the compositeness scale, we also have $\mu_{h \ell}^{0} \equiv \mu_{h \ell}(\mu \sim \Lambda) \sim \mathcal{O}\left(m_{W}\right)$. Therefore, since $\mu_{h / \ell}^{0} \equiv \mu_{h / \ell}(\mu \sim \Lambda) \sim \mathcal{O}(\Lambda)$, we expect $\left(\mu_{h \ell}^{0}\right)^{2} /\left(\mu_{h}^{0}\right)^{2} \sim \mathcal{O}\left(m_{W}^{2} / \Lambda^{2}\right) \ll 1$.

When the auxiliary (composite) field is integrated out at $\Lambda$, we recover the Lagrangian defined by Eq. 1 with

$$
G_{t^{\prime}}=\frac{\left(g_{t^{\prime}}^{0}\right)^{2}}{\left(\mu_{h}^{0}\right)^{2}}, G_{b^{\prime}}=\frac{\left(g_{b^{\prime}}^{0}\right)^{2}}{\left(\mu_{h}^{0}\right)^{2}}, G_{t^{\prime} b^{\prime}}=-\frac{g_{t^{\prime}}^{0} g_{b^{\prime}}^{0}}{\left(\mu_{h}^{0}\right)^{2}},
$$

plus additional interaction terms between the light Higgs and the new heavy quarks with Yukawa couplings of
$\mathcal{O}\left(\frac{\left(\mu_{h \ell}^{0}\right)^{2}}{\left(\mu_{h}^{0}\right)^{2}} \cdot g_{t^{\prime} / b^{\prime}}^{0}\right)$; since at $\Lambda$ we have $\left(\mu_{h \ell}^{0}\right)^{2} /\left(\mu_{h}^{0}\right)^{2} \sim$ $\mathcal{O}\left(m_{W}^{2} / \Lambda^{2}\right) \ll 1$, such residual $\Phi_{\ell} \bar{q}^{\prime} q^{\prime}$ terms are expected to be small and will, therefore, not participate in EWSB.

Thus, from the point of view of DEWSB, the hybrid $2 \mathrm{HDM}$ defined at $\Lambda$, i.e., with one fundamental and one auxiliary/composite scalar fields, is exactly equivalent to the theory defined in Eq. 1 with the strong 4-Fermi interactions of the 4th generation quarks. The auxiliary field, $\Phi_{h}$, is, therefore, viewed as a composite of the form $\Phi_{h} \sim g_{t^{\prime}}^{\star}<\bar{Q}_{L}^{\prime c}\left(i \tau_{2}\right) t_{R}^{\prime c}>+g_{b^{\prime}}<\bar{Q}_{L}^{\prime} b_{R}^{\prime}>$, which is responsible for EWSB and for the dynamical mass generation of the heavy quarks. At low energies the field $\Phi_{h}$ acquires a kinetic term as well as self interactions and the theory behaves as a $2 \mathrm{HDM}$ with a structure similar to the 4 G2HDM proposed in [16], i.e., one Higgs field $\left(\Phi_{h}\right)$ couples only to the heavy 4 th generation quarks and the 2 nd Higgs field $\left(\Phi_{\ell}\right)$ couples to the SM quarks of the 1st-3rd generations. The mass terms $\mu_{h}, \mu_{\ell}$ receive quantum corrections, resulting in EW-scale VEV's for $\Phi_{h}$ and for $\Phi_{\ell}$ which break the EW symmetry.

The resulting low-energy h4G2HDM scalar potential can be written as:

$$
\begin{aligned}
V_{h 4 G 2 H D M}\left(\Phi_{h}, \Phi_{\ell}\right) & =\mu_{\ell}^{2} \Phi_{\ell}^{\dagger} \Phi_{\ell}+\mu_{h}^{2} \Phi_{h}^{\dagger} \Phi_{h}-\mu_{h \ell}^{2}\left(\Phi_{h}^{\dagger} \Phi_{\ell}+\text { h.c. }\right) \\
& +\frac{1}{2} \lambda_{\ell}\left(\Phi_{\ell}^{\dagger} \Phi_{\ell}\right)^{2}+\frac{1}{2} \lambda_{h}\left(\Phi_{h}^{\dagger} \Phi_{h}\right)^{2}+\lambda_{3}\left(\Phi_{h}^{\dagger} \Phi_{h}\right)\left(\Phi_{\ell}^{\dagger} \Phi_{\ell}\right)+\lambda_{4}\left(\Phi_{h}^{\dagger} \Phi_{\ell}\right)\left(\Phi_{\ell}^{\dagger} \Phi_{h}\right)
\end{aligned}
$$

where all the above mass terms and quartic couplings run as a function of the energy scale $\mu$, as dictated by the RGE for this model. ${ }^{[4]}$ The stability condition for the above potential reads $\lambda_{\ell}, \lambda_{h}>0$ and $\sqrt{\lambda_{\ell} \lambda_{h}}>-\lambda_{3}-\lambda_{4}$. Also, we apply the compositeness boundary conditions at the scale $\Lambda$ to the strong 4th generation Yukawa couplings that generate the 4-Fermi terms and to the quartic couplings involving the auxiliary field $\Phi_{h}$ :

$$
\begin{aligned}
& g_{q^{\prime}}(\Lambda) \rightarrow \infty, \quad \lambda_{h, 3,4}(\Lambda) \rightarrow \infty, \\
& \lambda_{h}(\Lambda) / g_{q^{\prime}}^{4}(\Lambda) \rightarrow 0, \quad \lambda_{3,4}(\Lambda) / g_{q^{\prime}}^{2}(\Lambda) \rightarrow 0,
\end{aligned}
$$

where $q^{\prime}=t^{\prime}, b^{\prime}$. Eq. 7 reflects the fact that the h4G2HDM is matched at $\Lambda$ to the theory defined by Eq. 1. with the strongly coupled 4-Fermi factors derived

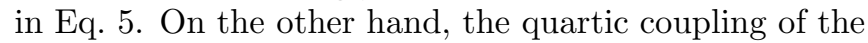
fundamental Higgs is unconstrained at $\Lambda$, so that we have $\lambda_{\ell}(\mu \rightarrow \Lambda) \rightarrow \lambda_{\ell}^{(0)}$, where $\lambda_{\ell}^{(0)}$ is a free parameter of the model.

[4] The most general 2HDM potential also includes the quartic couplings $\lambda_{5,6,7}$ [25], which, in our h4G2HDM, are absent at any scale.
The boundary conditions for $\mu_{h}, \mu_{\ell}$ and $\mu_{h \ell}$ are not required for our analysis, since we are only interested in their values at the EW-scale, which are fixed by the minimization conditions of the scalar-potential. In particular, at the minimum of the potential (i.e., at the EW-scale) we can express $\mu_{h}$ and $\mu_{\ell}$ in terms of $\mu_{h \ell}$, $\tan \beta \equiv t_{\beta}=v_{h} / v_{\ell}, v=\sqrt{v_{h}^{2}+v_{\ell}^{2}}$ and the quartic couplings $\lambda_{h}, \lambda_{\ell}$ (neglecting $\lambda_{3}$ and $\lambda_{4}$, see below):

$$
\mu_{\ell}^{2} \simeq \mu_{h \ell}^{2} / t_{\beta}-v^{2} c_{\beta}^{2} \lambda_{\ell} / 2, \quad \mu_{h}^{2} \simeq t_{\beta} \mu_{h \ell}^{2}-v^{2} s_{\beta}^{2} \lambda_{h} / 2,
$$

where $s_{\beta}, c_{\beta}=\sin \beta, \cos \beta$ and it is understood that the quartic couplings are evaluated at $\mu \sim v$, i.e., $\lambda_{h}=$ $\lambda_{h}(\mu \sim v)$ and $\lambda_{\ell}=\lambda_{\ell}(\mu \sim v)$.

\section{RESULTS AND DISCUSSION}

Solving the RGE of the h4G2HDM with the compositeness boundary conditions in Eq. 7, we find that $d \lambda_{3,4} / d \mu$ is proportional only to the (small) gauge couplings, so that $\lambda_{3}(v), \lambda_{4}(v)<<\lambda_{\ell}(v), \lambda_{h}(v)$ and can therefore be neglected. Also, in our h4G2HDM, the RGE for $\lambda_{\ell}$ and for the top-quark Yukawa coupling $g_{t}$ are similar to the SM RGE for these couplings. As for the RGE for $\lambda_{h}$ and 
for the Yukawa couplings of the 4th generation quarks $g_{q^{\prime}}(\mu)$, we can obtain a viable approximate analytic solution by neglecting the contributions from the running of the gauge couplings and the Yukawa couplings of all light fermions, as well as the Yukawa couplings of the 4th generation leptons. In particular, taking for simplicity $g_{t^{\prime}}=g_{b^{\prime}} \equiv g_{q^{\prime}}$, the dominant (approximate) RGE in our h4G2HDM are given by:

$$
\begin{aligned}
& \mathcal{D} g_{q^{\prime}} \approx 6 g_{q^{\prime}}^{3}, \\
& \mathcal{D} \lambda_{h} \approx 4 \lambda_{h}\left(3 \lambda_{h}+6 g_{q^{\prime}}^{2}\right)-24 g_{q^{\prime}}^{4},
\end{aligned}
$$

where $\mathcal{D} \equiv 16 \pi^{2} \mu \frac{d}{d \mu}$. With the compositeness boundary conditions of Eq. 7, the above RGE's have a simple analytic solution:

$$
g_{q^{\prime}}(\mu)=\sqrt{\frac{4 \pi^{2}}{3 \ln \frac{\Lambda}{\mu}}} \quad, \quad \lambda_{h}(\mu)=\frac{4 \pi^{2}}{3 \ln \frac{\Lambda}{\mu}} .
$$

Thus, using $m_{q^{\prime}}=v_{h} g_{q^{\prime}}\left(\mu=m_{q^{\prime}}\right) / \sqrt{2}$, we can obtain the cutoff $\Lambda$ as a function of $m_{q^{\prime}}$ and $t_{\beta}$ :

$$
\Lambda \approx m_{q^{\prime}} \cdot \exp \left(\frac{2 \pi^{2}\left(s_{\beta} v\right)^{2}}{3 m_{q^{\prime}}^{2}}\right) .
$$

In particular, for $m_{q^{\prime}}=400-600 \mathrm{GeV}$ and $t_{\beta} \sim$ $\mathcal{O}(1)$, for which our low-energy h4G2HDM successfully accounts for the recently discovered $125 \mathrm{GeV}$ Higgslike particle (see below), we find that $\Lambda \sim 1-1.5$ $\mathrm{TeV}$. Remarkably this is some fourteen orders of magnitudes smaller than the cutoff which emerges from a top-condensate scenario: $\Lambda \sim m_{t} \cdot \exp \left(\frac{16 \pi^{2} v^{2}}{9 m_{t}^{2}}\right) \sim 10^{17}$ $\mathrm{GeV}$, i.e., obtained by solving the SM-like RGE for $g_{t}$ : $\mathcal{D} g_{t} \approx \frac{9}{2} g_{t}^{3}$. Thus, introduction of a heavy quark doublet significantly alleviates the inherent fine-tuning (i.e., hierarchy) problem that afflicts the DEWSB models where the condensing fermions have masses $\lesssim 200 \mathrm{GeV} \cdot{ }^{[5]}$

The physical scalar masses are $m_{A}^{2}=$ $m_{H^{+}}^{2}=\mu_{h \ell}^{2} / s_{\beta} / c_{\beta}$ and $m_{h, H}^{2}=$ $\left(m_{1}^{2}+m_{2}^{2} \mp \sqrt{\left(m_{1}^{2}-m_{2}^{2}\right)^{2}+4 \mu_{h \ell}^{4}}\right) / 2$, where (see also Eq. (8):

$$
\begin{aligned}
& m_{1}^{2} \simeq \mu_{h}^{2}+3 s_{\beta}^{2} v^{2} \lambda_{h} / 2 \simeq t_{\beta} \mu_{h \ell}^{2}+s_{\beta}^{2} v^{2} \lambda_{h}, \\
& m_{2}^{2} \simeq \mu_{\ell}^{2}+3 c_{\beta}^{2} v^{2} / 2 \lambda_{\ell} \simeq \mu_{h \ell}^{2} / t_{\beta}+c_{\beta}^{2} v^{2} \lambda_{\ell},
\end{aligned}
$$

and a Higgs mixing angle:

$$
\tan 2 \alpha \simeq\left(\cot 2 \beta-v^{2}\left(s_{\beta}^{2} \lambda_{h}-c_{\beta}^{2} \lambda_{\ell}\right) / 2 / \mu_{h \ell}^{2}\right)^{-1},
$$

defined by $h=\cos \alpha \cdot \operatorname{Re}\left(\Phi_{\ell}^{0}\right)-\sin \alpha \cdot \operatorname{Re}\left(\Phi_{h}^{0}\right)$ and $H=$ $\cos \alpha \cdot \operatorname{Re}\left(\Phi_{h}^{0}\right)+\sin \alpha \cdot \operatorname{Re}\left(\Phi_{\ell}^{0}\right)$.

[5] For an interesting recent DEWSB model with three generations and $\Lambda \sim 10^{17}-10^{18} \mathrm{GeV}$, see [7].
Now, since the physical Higgs masses are sensitive to the energy scale at which the quartic couplings $\lambda_{h}$ and $\lambda_{\ell}$ are evaluated and since we do not apply the Higgs threshold corrections when solving the RGE, we need to estimate the errors on $m_{h}\left(\mu \sim m_{h}\right)$ and on $m_{H}(\mu \sim$ $\left.m_{H}\right)$. We do so by computing the difference between the masses obtained with $\lambda_{h / l}(\mu=100 \mathrm{GeV})$ and with $\lambda_{h / l}(\mu=400 \mathrm{GeV})$. We then find that the typical error is of $\mathcal{O}( \pm 10 \%)$ for $m_{h}$ and of $\mathcal{O}( \pm 20 \%)$ for $m_{H}$.

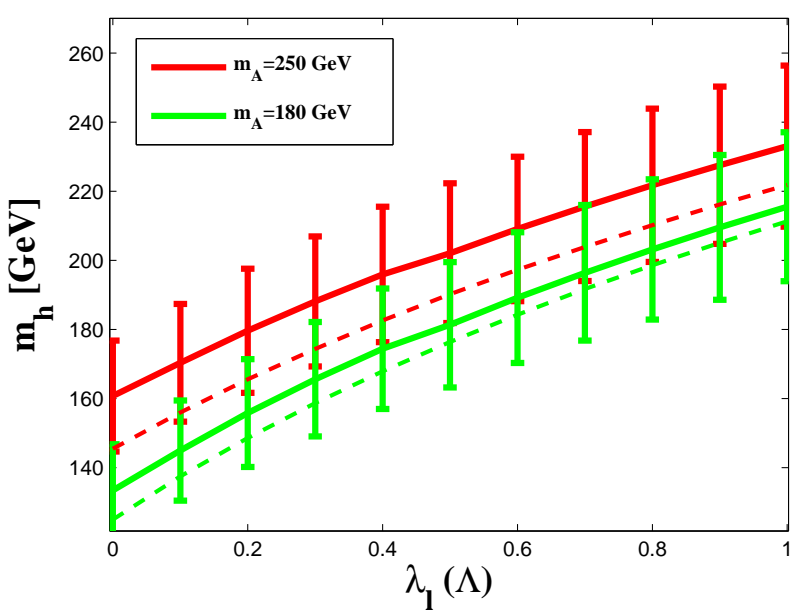

FIG. 1: $m_{h}$ as a function of $\lambda_{\ell}(\Lambda)$, for $\tan \beta=0.7, m_{q^{\prime}}=400$ GeV and two representative values of $m_{A}$. The approximate analytic solutions are shown by solid lines and exact results (obtained from a full RGE analysis, see text) without errors by the dashed lines.

As inputs we use $m_{A}$ (recall that $\left.\mu_{h \ell}=\sqrt{s_{\beta} c_{\beta}} m_{A}\right)$, $\tan \beta, v=246 \mathrm{GeV}, m_{q^{\prime}}$ (which sets the value of $\Lambda$, see Eq. 12) and $\lambda_{\ell}(\mu \sim \Lambda)$, while $\lambda_{h}(\mu)$ and $g_{q^{\prime}}(\mu)$ are calculated from Eq. 11. In Fig. 11 we show the typical dependence of $m_{h}$ on $\lambda_{\ell}(\Lambda)$, for $m_{q^{\prime}}=400 \mathrm{GeV}$ and for some representative values of $\tan \beta$ and $m_{A}$. We see that $m_{h}$ decreases with $\lambda_{\ell}(\Lambda)$, so that the minimal $m_{h}$ is obtained for $\lambda_{\ell}(\Lambda)=0$, i.e., at the boundary below which the vacuum becomes unstable. Note that, for $\lambda_{\ell}(\Lambda) \rightarrow 0$, we find $\lambda_{\ell}\left(m_{W}\right) \sim \mathcal{O}(0.1)$ and, in particular, $\lambda_{h}\left(m_{W}\right) \gg$ $\lambda_{\ell}\left(m_{W}\right)$. Thus, with $\lambda_{\ell}(\Lambda) \rightarrow 0$ and $\tan \beta \sim \mathcal{O}(1)$, we obtain $m_{h} \sim m_{A} / \sqrt{2}$ and $m_{H} \sim v \sqrt{\lambda_{h} / 2}$. It is also interesting to note that $\lambda_{\ell}(\Lambda) \rightarrow 0$ at the compositeness scale, raises the possibility that the "fundamental" scalar is a pseudo-Goldstone of some global symmetry breaking in the underlying strongly interacting theory, as in this case one expects a zero potential for $\Phi_{\ell}$ at tree-level with small loop corrections at energies below $\Lambda$.

In Fig. 2 we plot the minimal values of the lightest Higgs mass $m_{h}$ (i.e., the solutions obtained with $\lambda_{\ell}(\Lambda)=$ 0 ), as a function of $\tan \beta$, for $m_{q^{\prime}}=400 \mathrm{GeV}$ and $m_{A}=$ 180, 200 and $250 \mathrm{GeV}$. We note that the dependence of our results on $m_{q^{\prime}}$, or equivalently on $\Lambda\left(m_{q^{\prime}}\right)$, is negligible for values in the range $400 \mathrm{GeV} \lesssim m_{q^{\prime}} \lesssim 600 \mathrm{GeV}$, as long 

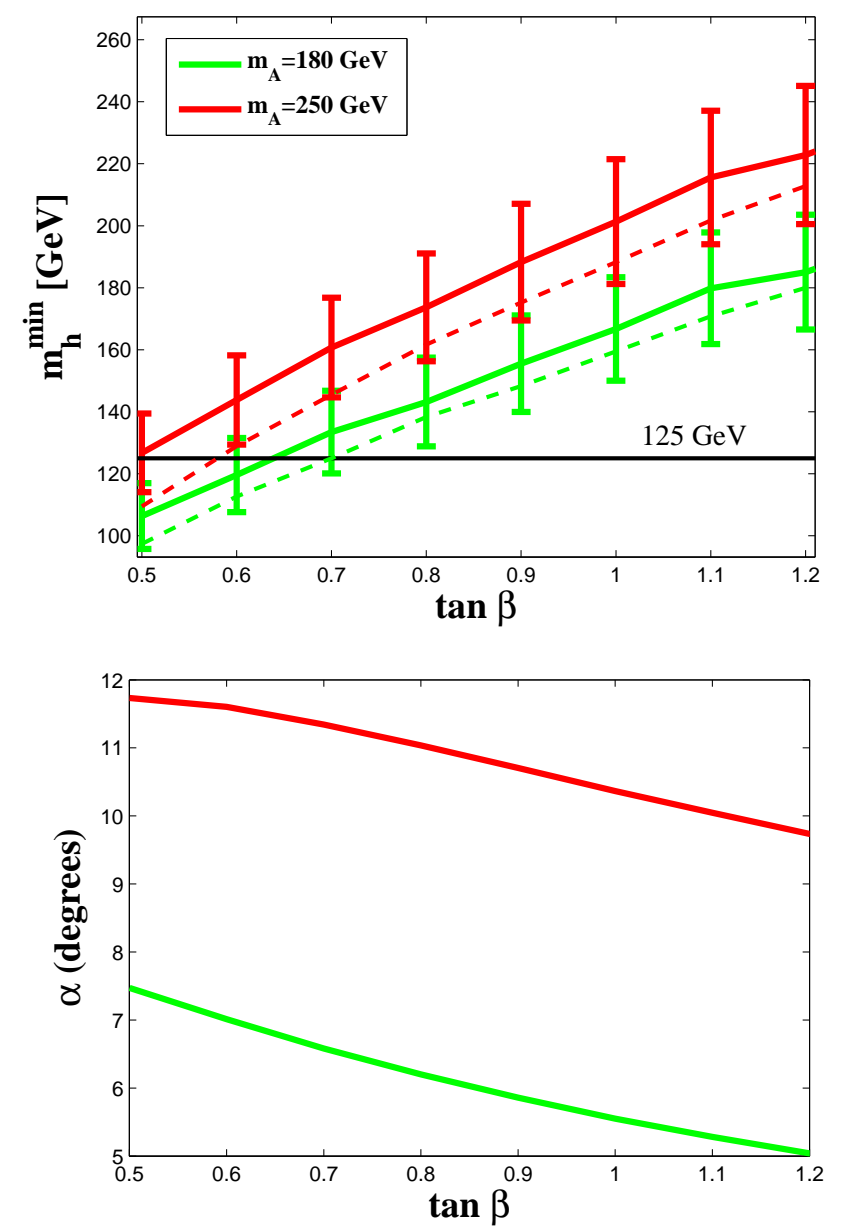

FIG. 2: The minimal value of $m_{h}$ (upper plot) and the corresponding values of the Higgs mixing angle $\alpha$ (lower plot), obtained by choosing $\lambda_{\ell}(\Lambda)=0$ (see text), as a function of $\tan \beta$ for $m_{A}=180$ and $250 \mathrm{GeV}$ and $m_{q^{\prime}}=600 \mathrm{GeV}$. See also caption to Fig. 1 .

as we choose the same boundary condition for $\lambda_{\ell}(\Lambda)$, i.e., at the compositeness scale.

Thus, we see that $m_{h} \sim 125 \mathrm{GeV}$ is obtained in the h4G2HDM with $\tan \beta \lesssim 0.7$ and $m_{A} \lesssim 250 \mathrm{GeV}$. This requires small values of $\lambda_{\ell}(\Lambda)$ (see Fig. (1) and a small Higgs mixing angle between the fundamental and the composite Higgs states of a typical size $\alpha \sim \mathcal{O}\left(10^{\circ}\right)$ (see Eq. 15 and Fig. (2). In particular, the light $125 \mathrm{GeV}$ Higgs state in our model is mostly the "fundamental" field, while the heavy CP-even Higgs is mostly a composite state. For the heavier Higgs we find that $m_{H} \sim 500 \pm 100 \mathrm{GeV}$ within the phenomenologically viable range of values of $m_{A}$ and $\tan \beta$, which give $m_{h} \sim 125 \mathrm{GeV}$.

We also depict in Figs. 1 and 2 the "exact" results, which are obtained from a full RGE analysis including the Yukawa couplings of the 4th generation leptons $\ell^{\prime}=\left(\nu^{\prime}, \tau^{\prime}\right)$ (assuming that $\nu^{\prime}$ and $\tau^{\prime}$ also couple to the auxiliary/composite field $\Phi_{h}$ and using $m_{\ell^{\prime}}=200$
$\mathrm{GeV}$ at the EW-scale), of the top and of the bottom quarks, as well as the gauge couplings. These agree quite well with our approximate calculations. Indeed, the observed slight shift from the approximate solutions is caused mainly by "turning on" the Yukawa couplings of the 4th generation leptons, and is within the estimated errors. We note, however, that the results are insensitive to the exact value of $m_{\ell^{\prime}}$, so long as it is within the range $\mathcal{O}\left(m_{W}\right)<m_{\ell^{\prime}}<\mathcal{O}\left(m_{q^{\prime}}\right)$.

\section{PHENOMENOLOGY: HIGGS SIGNALS AND CONSTRAINTS}

Before concluding, let us briefly discuss the collider phenomenology and the constraints from EW precision data (EWPD) on our model. As mentioned earlier, at the EW-scale, our model has the same spectrum and dynamics as the $4 \mathrm{G} 2 \mathrm{HDM}$ of [16]. We, therefore, apply the constraints from EWPD using the analysis in [16, 18], and we update the fit made in [20] to the $125 \mathrm{GeV}$ Higgs signals in this model. In particular, let us define the signal strength, which is the quantity usually being used for comparison between the measured and calculated (in a given model) Higgs signals:

$\mu_{X X}^{h 4 G 2 H D M(E x p)}=\frac{\sigma(p p \rightarrow h \rightarrow X X)_{h 4 G 2 H D M(E x p)}}{\sigma(p p \rightarrow h \rightarrow X X)_{S M}}$

and the individual pulls:

$$
\text { Individual pull }(\mathrm{XX}): \frac{\mu_{X X}^{h 4 G 2 H D M}-\mu_{X X}^{E x p}}{\sigma_{X X}},
$$

where for the observed ratios of cross-sections, i.e., the signal strengths $\mu_{X X}^{E x p}$, and the corresponding errors $\sigma_{X X}$, we use the latest results given in [26].

In Fig. 3 we plot the individual pulls in the h4G2HDM for the three most sensitive Higgs decay channels $p p \rightarrow$ $h \rightarrow W W^{*}, Z Z^{*}, \gamma \gamma$, as a function of the Higgs mixing angle $\alpha$ in the range applicable to the h4G2HDM setup (see Fig. 2) and for $\tan \beta=1$. In Table \we further list the resulting individual pulls in these three Higgs decay channels for values of $\tan \beta$ around $\tan \beta=1$ and for $\alpha=10^{\circ}, 20^{\circ}$. The rest of our model's parameter space is chosen such that:

1. It is consistent with EWPD: imposing the constraints from the oblique parameters $\mathrm{S}$ and $\mathrm{T}$, from $Z \rightarrow b \bar{b}$ and from B-physics $(b \rightarrow s \gamma$ and $B-\bar{B}$ mixing), following the analysis in [16, 18].

2. It is consistent with the results obtained above for our hybrid DEWSB scenario, i.e., with $m_{q^{\prime}} \sim$ $\mathcal{O}(500) \mathrm{GeV}, t_{\beta} \sim \mathcal{O}(1), m_{H} \sim \mathcal{O}(500) \mathrm{GeV}$ and $m_{A}=200-300 \mathrm{GeV}$ (see above).

In particular, the individual pulls in Fig. 3 and Table \ are calculated for the representative value $m_{t^{\prime}}=m_{b^{\prime}}=$ $600 \mathrm{GeV}$, where, for each value of $\alpha$ and $\tan \beta$, we also 
optimize (i.e., minimizing the $\chi^{2}$ for all Higgs channels) with respect to the parameter $\epsilon_{t}$, which, in our model, represents the mixing between the 4 th generation and the 3rd generation quarks (see also [16]). Furthermore:

- The individual pules are calculated for $m_{H^{+}}=200$ $\mathrm{GeV}$, but we have checked that they remain almost unchanged for larger $m_{H^{+}}$values.

- We assume that the Higgs is produced $100 \%$ through the 1-loop gluon-fusion mechanism.

- For the calculation of the Higgs production and decay vertices we use the latest version of Hdecay 27], with recent NLO contributions which also include the heavy 4th generation fermions, where we have inserted all the relevant couplings of our model.

- For the heavy lepton masses involved in the loops of the decays $h \rightarrow \gamma \gamma$ and in the 1-loop NLO corrections for the cases $h \rightarrow Z Z^{*}, W W^{*}$, we have used $m_{l^{\prime}}=m_{\nu^{\prime}}=200 \mathrm{GeV}$.

We see, that a good agreement with both the Higgs data and EWPD is obtained for $\alpha \sim 10^{0}$ and $\tan \beta \sim 1$ (we obtain $\chi^{2} \sim 5$ ), as required for the $125 \mathrm{GeV}$ hybrid Higgs state. As can be seen from Table【, our hybrid light Higgs is in very good agreement with the Higgs measurements in the $W W$ and $Z Z$ channels also for smaller (and larger) values of $\tan \beta$. On the other hand, there is a tension with the measurement in $p p \rightarrow h \rightarrow \gamma \gamma$ channel for $\tan \beta<1$. It should be noted, however, that the 1-loop $h \rightarrow \gamma \gamma$ vertex is expected to be sensitive to non-decoupling contributions of the new physics at the compositeness scale, which we cannot estimate without knowing the details of the physics at that scale. In this respect, the values of $\alpha$ and $\tan \beta$ (and for that matter also of the value of the compositeness scale $\Lambda$ ) predicted by our hybrid EWSB mechanism should not be taken at face value but rather as a guide (see also comment in the summary).

Finally, we note that our model can also lead to interesting new signatures of the heavy quarks, such as the flavor changing decay $t^{\prime} \rightarrow t h$, which can be searched for in the present LHC data using a specific search strategy, see [19]. Indeed, very recently the ATLAS collaboration has performed a search for $t^{\prime}$ of an $\mathrm{SU}(2)$ doublet, using selection criteria designed for the case in which $t^{\prime} \rightarrow t h$ followed by $h \rightarrow \bar{b} b$ [28]. They obtained a new bound $m_{t^{\prime}} \gtrsim 800 \mathrm{GeV}$, which is appreciably stronger than the previously existing bound (see [29]). This new bound is in tension with our DEWSB framework, since such a heavy 4th generation doublet, if it is chiral, will be non-perturbative at energy scales already smaller than its mass. ${ }^{[6]}$ We emphasized, however,

[6] We note, however, that the recent search performed by ATLAS in [28] assumes $t^{\prime} \rightarrow t h$ followed by $h \rightarrow b \bar{b}$, while in our low

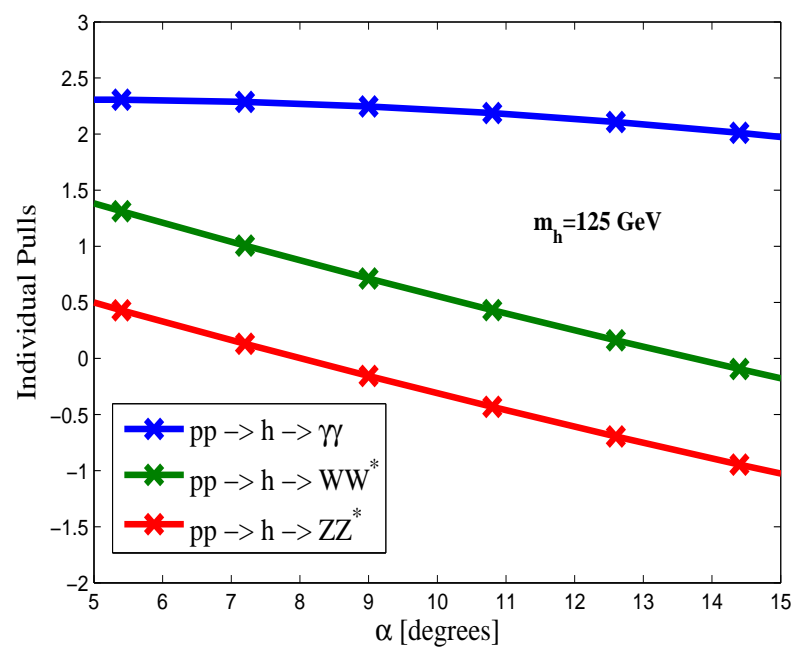

FIG. 3: The individual pulls as defined in Eq. 17, in the channels $p p \rightarrow h \rightarrow W W^{*}, Z Z^{*}, \gamma \gamma$, as a function of mixing angle $\alpha$ and for: $\tan \beta=1, m_{t^{\prime}}=m_{b^{\prime}}=600 \mathrm{GeV}$, $m_{l_{4}}=m_{\nu_{4}}=200 \mathrm{GeV}, m_{H^{+}} \geq 200 \mathrm{GeV}$ (see text), and optimizing with respect to $\epsilon_{t}$ (see text). All points are in agreement with the EWPD.

\begin{tabular}{|c|c|c|c|c|c|c|c|}
\hline $\tan \beta \Rightarrow$ & 0.7 & 0.8 & 0.9 & 1 & 1.1 & 1.2 & 1.3 \\
\hline$\alpha=10^{0}$ & -0.3 & 0.04 & 0.3 & 0.5 & 0.7 & 0.8 & 0.8 \\
\hline$\alpha=20^{0}$ & -1.8 & -1.6 & -1.5 & -1.5 & -1.4 & -1.4 & -1.5 \\
\hline \multicolumn{8}{|c|}{ individual pulls for $p p \rightarrow h \rightarrow Z Z^{\star}$} \\
\hline $\tan \beta \Rightarrow$ & 0.7 & 0.8 & 0.9 & 1 & 1.1 & 1.2 & 1.3 \\
\hline$\alpha=10^{0}$ & -1.1 & -0.7 & -0.4 & -0.2 & -0.01 & 0.1 & 0.2 \\
\hline$\alpha=20^{\circ}$ & -2.8 & $|-2.6|$ & $-2.5 \mid$ & -2.4 & -2.4 & $|-2.4|$ & -2.5 \\
\hline \multicolumn{8}{|c|}{ individual pulls for $p p \rightarrow h \rightarrow \gamma \gamma$} \\
\hline $\tan \beta \Rightarrow$ & 0.7 & 0.8 & 0.9 & 1 & 1.1 & 1.2 & 1.3 \\
\hline$\alpha=10^{0}$ & 73.4 & 34.2 & 13.3 & 1.9 & \begin{tabular}{|l|} 
\\
-2.7
\end{tabular} & -2.4 & -1.9 \\
\hline$\alpha=20^{0}$ & 7.1 & -2.9 & -7.0 & -6.7 & \begin{tabular}{l|l}
7 & -6.6
\end{tabular} & -6.8 & -7.1 \\
\hline
\end{tabular}

TABLE I: The individual pulls as defined in Eq. 17, in the channels $p p \rightarrow h \rightarrow W W^{*}, Z Z^{*}, \gamma \gamma$, for $\alpha=10^{0}$ and $20^{\circ}$ and for values of $\tan \beta$ in the range $\tan \beta=1 \pm 0.3$. The rest of the parameters are as in Fig. [ and all points are in agreement with the EWPD.

that our hybrid DEWSB framework can be equally constructed with $\mathrm{TeV}$-scale vector-like quarks [15]. In such a case, our hybrid mechanism can be applied also with a higher compositeness scale of several $\mathrm{TeV}$, without being in conflict with perturbativity of the vector-like quarks Yukawa couplings. We leave these issues for a future

energy h4G2HDM, $h \rightarrow g g$ might be the dominant decay, see [20]. 
work.

\section{SUMMARY}

To summarize, we have constructed a hybrid scenario for DEWSB, where both a fundamental-like Higgs field and a condensate of a strongly coupled heavy quark sector participate in EWSB. This yields a partly dynamical EWSB setup, and the resulting low-energy theory is a hybrid 2HDM with another family of heavy fermions. In particular, in this model one Higgs (mostly fundamental) couples only to the light SM fermions while the 2nd Higgs (mostly composite) couples only to the heavy (dynamical) new fermions. The proposed DEWSB framework results in a compositeness scale $\Lambda \sim \mathrm{TeV}$ and a phenomenologically viable low energy setup, which closely resembles the recently proposed model of [16 and, as we show, is consistent with current EW precision and flavor data. In particular, in spite of the heaviness of the dynamical quarks, $m_{q^{\prime}} \sim \mathcal{O}(500) \mathrm{GeV}$, and the resulting low TeV-scale threshold for the strong dynamics, a viable Higgs candidate is obtained, which has a mass $m_{h} \sim 125 \mathrm{GeV}$ and properties very similar to the SM Higgs, when $\tan \beta \sim \mathcal{O}(1), m_{A} \sim m_{H^{+}} \sim 200-300 \mathrm{GeV}$ and $m_{H} \sim \mathcal{O}(500) \mathrm{GeV}$. Indeed, we perform a fit to the recently measured $125 \mathrm{GeV}$ Higgs signals, also imposing the constraints from precision EW data, and demonstrate the consistency of our model with all current collider phenomenology.
We emphasize that the purpose of this work is to present a mechanism that addresses the low-energy outcome of a possible $\mathrm{TeV}$-scale strong sector and not to discuss the details of the strongly interacting theory. Indeed, we show that this mechanism (the hybrid structure of DEWSB) is very predictive without knowing the details of the physics at the cutoff scale. In this respect, the exact values obtained for the free-parameters of the model, i.e., for $m_{A}, \tan \beta$ and $\Lambda$, have no deep meaning, but should rather be interpreted as a guide. For example, the exact position of a Landau pole cannot be taken at face value, since the threshold effects are unknown without knowing the details of the UV completion of the model. In particular, the expression we get for $\Lambda$ in Eq. 12 is an estimate from the RGE of the model and it is valid only in the range where the RGE are valid and can be used in a perturbative manner. Clearly, this is not the case as one approaches the compositeness scale. For that reason it is fair to say that $\Lambda \sim 1 \mathrm{TeV}$ should be rather viewed as $\Lambda \sim$ few TeV. Similarly, $\tan \beta \sim 0.7$ should be interpreted as $\tan \beta \sim \mathcal{O}(1)$ and the value we get for the Higgs mixing angle, i.e., $\alpha \sim \mathcal{O}\left(10^{0}\right)$ should be taken as a guide.

Acknowledgments: SBS and MG acknowledge research support from the Technion. The work of AS was supported in part by DOE contract \#DE-AC0298CH10886(BNL).
[1] S. Chatrchyan et al., [CMS Collaboration], Phys. Lett. B716, 30 (2012); G. Aad et al., [ATLAS Collaboration], Phys. Lett. B716, 1 (2012).

[2] S. Weinberg, Phys. Rev. D19, 1277 (1979); L. Susskind, Phys. Rev. D20, 2619 (1979). See also, R. Jackiw and K. Johnson, Phys. Rev. D8, 2386 (1973); J.M. Cornwall and R.E. Norton, Phys. Rev. D8, 3338 (1973).

[3] Y. Nambu and G. Jona-Lasinio Phys. Rev. 122, 345 (1961).

[4] V.A. Miransky, M. Tanabashi and K. Yamawaki, Phys. Lett. B221, 177 (1989); ibid., Mod. Phys. Lett. A4, 1043 (1989); W.A. Bardeen, C.T. Hill and M. Lindner, Phys. Rev. D41, 1647 (1990); see also Y. Nambu in e.g., EFI report \#89-08, 1989 (unpublished).

[5] G. Burdman, C.E.F. Haluch, JHEP 1112, 038 (2011); B. Holdom, Phys. Lett. B721, 290 (2013).

[6] C.T. Hill, Phys. Lett. B345, 483 (1995).

[7] A. Smetana, Eur. Phys. J. C73, 2513 (2013).

[8] B. Holdom, Phys. Rev. Lett. 57, 2496 (1986), Erratumibid. 58, 177 (1987); S.F. King, Phys. Lett. B234, 108 (1990); P.Q. Hung and G. Isidori Phys. Lett. B402, 122 (1997); B. Holdom, JHEP 0608, 76 (2006); Y. Mimura, W.S. Hou and H Kohyama, JHEP 1311, 048 (2013).

[9] M.A. Luty, Phys. Rev. D41, 2893 (1990).

[10] C. Hill, M. Luty and E.A. Paschos, Phys. Rev. D43, 3011 (1991).

[11] G. Burdman and L. Da Rold, JHEP 0712, 86 (2007).
[12] P.Q. Hung, C. Xiong, Nucl. Phys. B848, 288 (2011); for an earlier discussion see also, "Dynamical symmetry breaking due to strong coupling Yukawa interaction" by M. Tanabashi, K. Yamawaki and K. Kondo, in Nagoya 1989, Proceedings, Dynamical symmetry breaking 28-36.

[13] G. Burdman, L. Da Rold, O. Eboli and R.D. Matheus, Phys. Rev. D79, 075026 (2009); M. Hashimoto and V.A. Miransky, Phys. Rev. D81, 055014 (2010); A.E.C. Hernandez, C.O. Dib, H.N. Neill and A.R. Zerwekh, JHEP 1202, 132 (2012); P.Q. Hung, C. Xiong, Nucl. Phys. B847, 160 (2011); P.Q. Hung, C. Xiong, Phys. Lett. B694, 430 (2011); C.M. Ho, P.Q. Hung and T.W. Kephart, JHEP 1206, 45 (2012). G. Burdman, L. de Lima and R.D. Matheus, Phys. Rev. D83, 035012 (2011).

[14] B. Chung, K.Y. Lee, D.-W. Jung and P. Ko, JHEP 0605, 010 (2006).

[15] See e.g., N. Arkani-Hamed, A.G. Cohen, H. Georgi, Phys. Lett. B513, 232 (2001); N. Arkani-Hamed, A. Cohen, E. Katz, A. Nelson, JHEP 0207, 034 (2002); M. Perelstein, Prog. Part. Nucl. Phys. 58, 247, 2007); R. Contino, L.D. Rold, A. Pomarol, Phys. Rev. D75, 055014 (2007); M. Carena, E. Ponton, J. Santiago, C.E. Wagner, Nucl. Phys. B759, 202 (2006); J.A. Aguilar-Saavedra, JHEP 11, 030 (2009).

[16] S. Bar-Shalom, S. Nandi and A. Soni, Phys. Rev. D84, 053009 (2011).

[17] For a recent analysis see, O. Eberhardt et. al., Phys. Rev. 
Lett. 109, 241802 (2012).

[18] S. Bar-Shalom, M. Geller, S. Nandi and A. Soni, arXiv:1208.3195 [hep-ph], a review published in a special issue of Advances in High Energy Physics (AHEP) on "Very Heavy Quarks at the LHC", Adv.High Energy Phys.2013, 672972 (2013).

[19] M. Geller, S. Bar-Shalom and G. Eilam, Phys. Lett. B715, 121 (2012).

[20] M. Geller, S. Bar-Shalom, G. Eilam and A. Soni, Phys. Rev. D86, 115008 (2012).

[21] M. Hashimoto, Phys. Rev. D81, 075023 (2010); H.-S. Lee and A. Soni, Phys. Rev. Lett. 110, 021802 (2013); M. Sher, Phys. Rev. D61, 057303 (2000); L. Bellantoni, J Erler, J. Heckman and E. Ramirez-Homs, Phys. Rev. D86, 034022 (2012); W. Bernreuther, P. Gonzales and M. Wiebusch, Eur. Phys. J. C69, 31 (2010); John F. Gunion, arXiv:1105.3965 [hep-ph]; X.-G. He and G. Valencia, Phys. Lett. B707, 381 (2012); N. Chen and H. He, JHEP 1204, 062 2012; E. De Pree, G. Marshall and M. Sher, Phys. Rev. D80, 037301 (2009); M. S. Chanowitz,
arXiv:1212.3209 [hep-ph].

[22] C.T. Hill, Phys. Lett. B266, 419 (1991).

[23] "Top mode standard model" by K. Yamawaki, in Strong Coupling Gauge Theories and beyond (SCGT 90), Proceedings 28-31, Jul 1990, Nagoya, Japan.

[24] R.D. Peccei and H.R. Quinn, Phys. Rev. D16, 1791 (1977).

[25] G.C Branco et. al., Phys. Rept. 516 (2012).

[26] See e.g., "Higgs bosons in the SM and beyond", talk given by G. Landsberg at The 2013 European Physical Society Conference on High Energy Physics, EPSHEP 2013, Stockholm, Sweden, 18-24 July, 2013.

[27] A. Djouadi, J. Kalinowski and M. Spira, Comput. Phys. Commun. 108, 56 (1998), with contributions from arXiv:hep-ph/9704448 [hep-ph].

[28] ATLAS Collaboration, ATLAS-CONF-2013-018, ATLAS-COM-CONF-2013-024.

[29] Georges Aad (Freiburg U.) et al. (ATLAS Collaboration), Phys. Lett. B718, 1284 (2013). 